\section{Reduction environmental effects of civil aircraft through multi-objective flight plan optimisation}

To cite this article: D S Lee et al 2010 IOP Conf. Ser.: Mater. Sci. Eng. 10012197

View the article online for updates and enhancements.

\section{Related content}

The Combined Multi-objective Optimization Design for a Light Guide Rod Yu-Sen Yang, Chun-Yao Shih, Hong-Yao Chien et al.

Optimal locations of piezoelectric patches for supersonic flutter control of honevcomb sandwich panels, using the NSGA-II method

M Nezami and B Gholami

Multi-objective constrained design of nickel-base superalloys using data miningand thermodynamics-driven genetic algorithms

Edern Menou, Gérard Ramstein,

Emmanuel Bertrand et al. 


\title{
Reduction Environmental Effects of Civil Aircraft through Multi-Objective Flight Plan Optimisation
}

\author{
D S Lee ${ }^{1}$, L F Gonzalez ${ }^{2}$, R Walker ${ }^{2}$, J Periaux $^{3}$ and E. Onate ${ }^{3}$ \\ ${ }^{1}$ International Center for Numerical Methods in Engineering (CIMNE/UPC), Edificio \\ C3, Room 216, Parque Mediterraneo de la Tecnologia, C/Esteve n. 508860 \\ Castelldefels, Spain. \\ ${ }^{2}$ Australian Research Centre for Aerospace Automation (ARCAA), Queensland \\ University of Technology (QUT), Brisbane, QLD 4001 Australia. \\ ${ }^{3}$ International Center for Numerical Methods in Engineering (CIMNE/UPC), Edificio \\ C1, Gran Capitan s/n. 08034 Barcelona, Spain. \\ E-mail: ds.chris.lee@gmail.com
}

\begin{abstract}
With rising environmental alarm, the reduction of critical aircraft emissions including carbon dioxides $\left(\mathrm{CO}_{2}\right)$ and nitrogen oxides (NOx) is one of most important aeronautical problems. There can be many possible attempts to solve such problem by designing new wing/aircraft shape, new efficient engine, etc. The paper rather provides a set of acceptable flight plans as a first step besides replacing current aircrafts. The paper investigates a green aircraft design optimisation in terms of aircraft range, mission fuel weight $\left(\mathrm{CO}_{2}\right)$ and NOx using advanced Evolutionary Algorithms coupled to flight optimisation system software. Two multi-objective design optimisations are conducted to find the best set of flight plans for current aircrafts considering discretised altitude and Mach numbers without designing aircraft shape and engine types. The objectives of first optimisation are to maximise range of aircraft while minimising NOx with constant mission fuel weight. The second optimisation considers minimisation of mission fuel weight and NOx with fixed aircraft range. Numerical results show that the method is able to capture a set of useful trade-offs that reduce $\mathrm{NOx}$ and $\mathrm{CO}_{2}$ (minimum mission fuel weight).
\end{abstract}

\section{Introduction}

Emissions targets worldwide and climatic effects have put pressure in government agencies, aircraft manufacturers and airlines to reduce water vapour, carbon dioxide $\left(\mathrm{CO}_{2}\right)$ and oxides of nitrogen $(\mathrm{NOx}$ $=\mathrm{NO}+\mathrm{NO}_{2}$ ) resulting from aircraft emissions [1-4]. The major, large-scale environmental problem associated with the continuing expansion of aviation is the forcing of climate change. During flight, aircraft engines emit carbon dioxide, oxides of nitrogen, oxides of sulphur, water vapour, hydrocarbons and sulphur oxide particles. These emissions alter the chemical composition of the atmosphere in a variety of ways, both directly and indirectly. Many of the emissions from aircraft change the absorption of solar radiation and the absorption and emission of thermal radiation. They may, therefore, affect climate. Important aspects of such climate change could be a local change in average precipitation or the frequency and intensity of heat waves. It is thought that new regulations on permitted levels of oxides of nitrogen may limit the expansion of some airports. The Kyoto

1 To whom any correspondence should be addressed. 
Protocol places limits only on carbon dioxide emissions, not the emissions of oxides of nitrogen and water vapour. These gases, when emitted at high altitudes, cause more damage than they do at ground level, resulting in greater 'radiative forcing' from aviation than might be expected from its carbon dioxide emissions alone [2]. Oxides of nitrogen, for instance, produced by high temperature burning in the engine, are rapidly involved in chemical reactions that lead to changes in both ozone and ambient methane.

This is a multi- disciplinary problem with multiple trade-offs such as maximising range, minimising mission fuel weight, minimise emissions while maintaining aircraft separation and air safety.

Research studies and mathematical models have shown that an optimised flight plan combined with advanced separation management strategies can reduce carbon dioxide and oxides of nitrogen. Multidisciplinary approach using advanced Multi-Objective Evolutionary Algorithm (MOEA) can be used to extend the range of an aircraft and reduce the fuel consumption and NOx without compromising on aircraft geometry and engine types.

Even though there are a number of models for water vapour, carbon dioxide and oxides of nitrogen reduction, most consider flight plan optimisation. This research will develop a multidisciplinary algorithm which takes into account aircraft performance, flight plan optimisation and also develop mathematical model and algorithms for carbon dioxide and oxides of nitrogen reduction through flight optimisation.

The rest of this report is organised as follows; the description of the analysis tool and method is given in Section 2. The analysis and formulation of design problem is described in sections 3. The applications of the method to real world problems and optimisation studies are shown in Section 4. Finally, Section 5 contains conclusions and directions for future research.

\section{Method and Analysis Tool}

The approach used in this research consisted on first selecting a typical commercial aircraft and conduct a full analysis of range, fuel consumption and aircraft emissions using flight optimisation system coupled to advanced Multi-Objective Evolutionary Optimiser MOEA. The MOEA used in this is called HAPMOEA developed by authors.

\subsection{Analysis tool}

This project used the flight optimisation system (FLOPS) code to analyse mission performance of a generic commercial aircraft [5]. FLOPS developed by NASA is a multidisciplinary system software for preliminary and conceptual design. It has nine primary modules to evaluate advanced aircraft concepts including weights, aerodynamics, engine cycle analysis, propulsion data scaling and interpolation, mission performance, take-off and landing, noise footprint, cost analysis and program control.

In this project, five modules within FLOPS are including weights, aerodynamics, engine cycle, propulsion data scaling and interpolation and mission performance. FLOPS uses statistical/empirical equations to predict the weight of whole aircraft and also it applies a modified version of the Empirical Drag Estimation Techniques (EDET). The module for engine cycle are developed by Geiselhart [6 -8] and has capabilities to generate an engine deck consisting of thrust and fuel flow data at a given Mach and altitude conditions. This engine deck from engine cycle module will be used by propulsion data scaling and interpolation to produce propulsion data for mission performance. The mission performance module uses the calculated weights, aerodynamics and propulsion data to calculate mission fuel weight, range, endurance and NOx emissions at a given flight conditions. Details of FLOPS can be found in Reference $[5,16]$. In this analysis, FLOPS calculates either the range of aircraft or weight when ramp weight or mission range is fixed. 


\subsection{Hierarchical Asynchronous Parallel Multi-Objective Evolutionary Algorihtms (HAPMOEA)}

The optimisation algorithm used in this project is based on Evolution Strategies [9 -11] and incorporates to the concepts of Covariance Matrix Adaptation (CMA) [12], Distance Dependent Mutation (DDM), a hierarchical topology, asynchronous evaluation and a Pareto tournament selection that is applicable to single or multi-objective problems. The hierarchical topology can provide different models including precise, intermediate and approximate models. Each node belonging to the different hierarchical layer can be handled by a different EAs code. Details of HAPMOEA can be found in reference [13-15].

In this project, HAPMOEA is updated to handle both real and binary coding to optimise discretised design variables due to the operating conditions (Mach and altitude) optimisation for example the optimised Mach number and altitude will become 0.7 or 0.75 (discretised step 0.5) 29,000 or 30,500 ft (discretised step $500 \mathrm{ft}$ ) respectively. The discretised step can be defined by user.

\subsection{Optimisation algorithm coupled analysis tool}

To couple analysis tool FLOPS and multi-objective optimiser HAPMOEA, the interface software was developed. This interface software obtains a set of design information from HAPMOEA and generates input file which is readable format for FLOPS. It will collect mission performance data and transfer to HAPMOEA which will compute fitness functions.

Figure 1 shows the flow chart algorithm developed in this project where there are eight major steps.

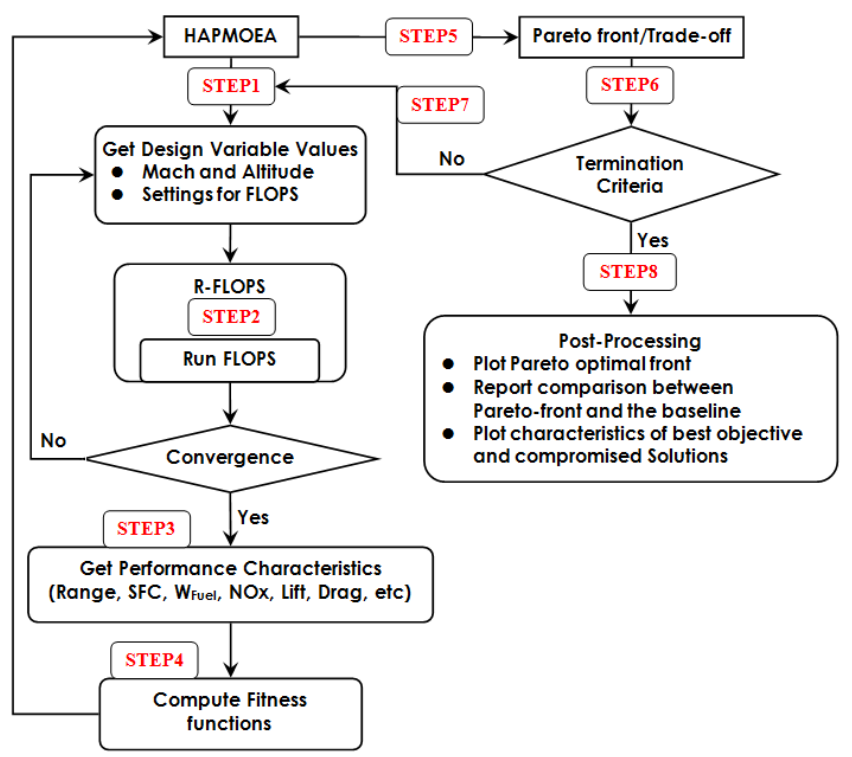

Figure 1. Overall optimisation.

Step 1. Obtain constant aircraft data, design bounds and optimisation settings and generate design variables information file which includes operating conditions, constant aircraft configurations.

Step 2. Run interface code R-FLOPs and generate input file for analysis tool FLOPS.

Step 3. Obtain performance data including range, Specific Fuel Consumption, mission fuel weight $\left(W_{F u e l}\right), \mathrm{NOx}$, lift, drag, etc and plot performance data.

Step 4. Compute fitness functions and send back to HAPMOEA.

Step 5. Plot Pareto optimal solutions.

Step 6. Check the termination/stopping criteria.

Step 7. Repeat from Step 1 to Step 6 when Step 6 is "No".

Step 8. Post-Processing including plotting Pareto optimal front and their mission characteristics, and generating comparison report between the baseline and Pareto optimal solutions. 


\section{Analysis and Formulation of Design Problem}

The problem considers a typical 189 passengers jet aircraft which is similar to Boeing 737-800. The baseline aircraft specifications are obtained from references $[17,18]$ and are indicated in Table 1 . The coordinates of the baseline aerofoil sections at four sections (root, crank1, crank2 and tip) are obtained from reference [19] as shown Figure 2.

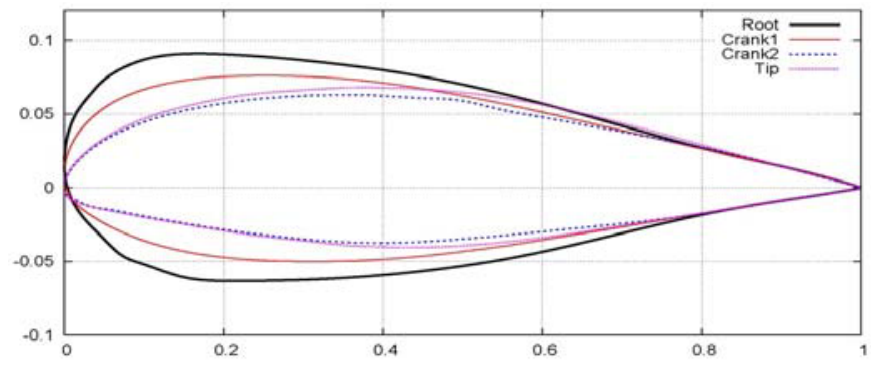

Figure 2. Baseline wing aerofoil sections.

Table 1. Baseline aircraft specifications.

\begin{tabular}{ccc}
\hline & Parameters & Values $\left(f t, f t^{2}, l b\right)$ \\
\hline Fuselage & Length & 129.6 \\
& Width & 12.4 \\
& Height & 13.2 \\
\hline Wing & Dihedral effect & $6^{\circ}$ \\
& Span & 117.5 \\
& Area & 1341 \\
& $A R$ (Aspect Ratio) & 9.45 \\
& Trapezoidal Root chord length, $t / c$ & $18.7,0.16$ \\
& Tip chord length, $t / c$ & $4.1,0.105$ \\
& Average ratio of $t / c$ & 0.1225 \\
& Taper ratio & 0.159 \\
& $1 / 4$ chord sweep angle & $25.02^{\circ}$ \\
& Ratio of Flap area to Wing area & 0.3 \\
\hline Horizontal & Area & 352.8 \\
Stabilizer & 1/4 chord sweep angle & $30^{\circ}$ \\
& $A R$ & 6.16 \\
& Taper ratio & 0.203 \\
\hline Vertical & Area & 284.6 \\
Stabilizer & 1/4 chord sweep angle & $35^{\circ}$ \\
& $A R$ & 1.91 \\
& Taper ratio & 0.271 \\
\hline
\end{tabular}

The baseline aircraft uses two CFM56-7B27 turbofan engines $[19,20]$. The specifications of this engine are shown in Table 2. This engine allows the baseline aircraft to flight at maximum altitude $41,000 \mathrm{ft}$ with the maximum cruise speed of Mach 0.82 . The typical altitude and cruise Mach number are $35,000 f t$ and 0.785 respectively.

Table 1. CFM56-7B27 specifications.

\begin{tabular}{ll}
\hline Descriptions & Values \\
\hline Max. Thrust & $27,300 \mathrm{lb}$ \\
Bypass ratio & 5.1
\end{tabular}




$\begin{array}{ll}\text { Overall pressure ratio } & 32.8 \\ \text { Length } & 98.7 \mathrm{in} \\ \text { Fan diameter } & 61.0 \mathrm{in} \\ \text { Basic dry weight } & 5,216 \mathrm{lb} \\ \text { Applications } & 737-800 / 900 \text { and BBJ }\end{array}$

\subsection{Baseline Aircraft Analysis}

In this research, we use the flight optimisation system FLPOS. The data in Table 2 is used as input parameters for FLOPS to analyse the weight and specific fuel consumption. Table 3 compares the output obtained by FLOPS and published data [21]. It can be seen that there is very good agreement between the outputs obtained by FLOPS and published data.

Table 2. Comparison weight distribution obtained by FLOPS and public data.

\begin{tabular}{llll}
\hline Parameters & Real Data & FLOPS & Deviation \\
\hline Fuel Weight $(l b)$ & 46,305 & 45,080 & $-2.6 \%$ \\
Empty Weight $(l b)$ & 82,535 & 82,575 & $+0.05 \%$ \\
Operating Empty Weight $(l b)$ & 90,710 & 89,901 & $-0.89 \%$ \\
Payload $(l b)$ & 40,059 & 39.690 & $-0.9 \%$ \\
SFC $(l b)$ & 0.627 & 0.637 & $+1.6 \%$ \\
\hline
\end{tabular}

From this analysis, it is seen that the baseline aircraft can flight 3,485 $\mathrm{nm}$ at 35,000 $\mathrm{ft}$ with Mach 0.785 while producing $307 \mathrm{lb}$ of the oxides nitrogen (NOx) after consuming 45,080 lb of mission fuel. The analysed range $(3,485 \mathrm{~nm})$ is $12 \%$ longer than the real data $(3,060 \mathrm{~nm})$.

\section{Real-World Design Optimisation}

In this section, two optimisations are conducted without reconfiguring aircraft geometry or engine type; the first optimisation with fixed ramp weight is to maximise the range of the baseline aircraft while minimising NOx. The second optimisation with fixed cruise range is to minimise the mission fuel weight $\left(\mathrm{CO}_{2}\right)$ while minimising NOx emission.

\subsection{Optimisation - I: Fixed Ramp Weight}

\subsubsection{Problem Definition}

This optimisation is to find the best Mach and altitude operating conditions which allows the aircraft to have maximum range and minimum NOx with fixed ramp weight $(174,670 \mathrm{lb})$. The fitness functions are defined by equations (4) and (5);

$$
\begin{gathered}
f_{1}=\min \left(W_{\text {Fuel }} / \text { Range }\right) \\
f_{2}=\min (N O x)
\end{gathered}
$$

with the following discretised design bounds

$$
M_{\infty} \in[0.7: 0.005: 0.82] \text { and Altitude } \in[29,000: 500 f t: 41,000] .
$$

where mission fuel weight $\left(W_{\text {Fuel }}\right)$ is constant. For the design, there are 24 discretised Mach numbers and altitudes with step size 0.005 and $500 \mathrm{ft}$ respectively.

\subsubsection{Numerical Results}

The optimisation ran for 5 hours using one CPU $4 \times 2.8 \mathrm{GHz}$. Pareto optimal solutions are illustrated in Figure 3 where all Pareto members produce lower NOx (fitness function 2) when compared to the baseline operating conditions $\left(M_{\infty}=0.785\right.$, Altitude $\left.=35,000 f t\right)$. However Pareto members 9 to 20 are 
dominated by the baseline operating conditions in terms of range (fitness function 1) as shown in Table 4. It can be seen that Pareto member 20 has only $2 \%$ lower range when compared to the baseline design. Pareto members 1 to 8 can be selected as compromised solutions since they have higher range and lower NOx when compared to the baseline operating conditions. However the design engineers could choose one of the operating conditions obtained by Pareto members 9 to 20 due to NOx reduction if there is a regulation for NOx emission at the cruise conditions.

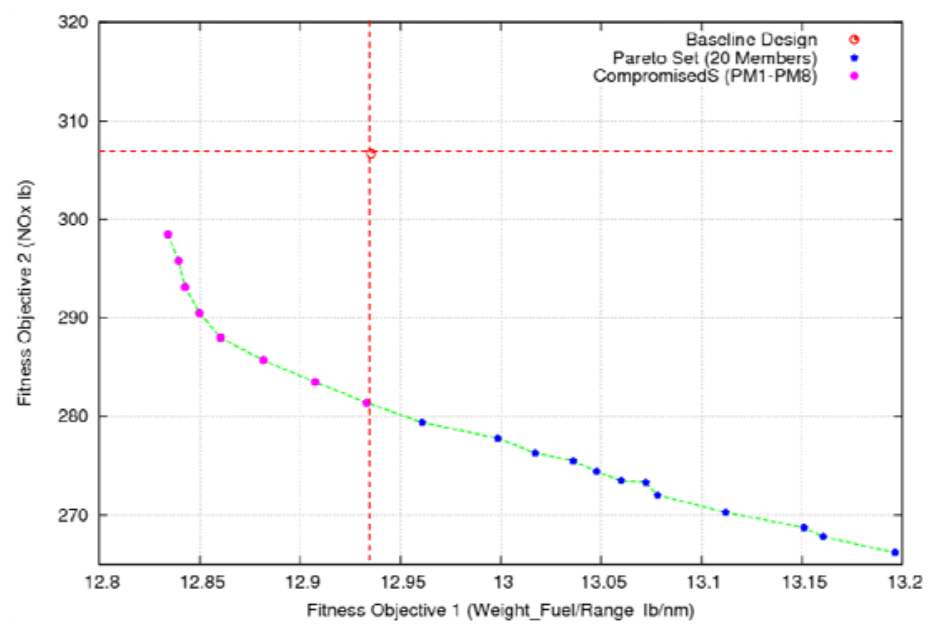

Figure 3. Pareto optimal front for NOx vs. $\mathrm{W}_{\text {Fuel }} /$ Range at fixed ramp weight.

Table 4. Mission Cruise Range and NOx obtained by optimal solutions and baseline design.

\begin{tabular}{|c|c|c|c|c|c|c|}
\hline Models & Mach & Altitude $(f t)$ & SFC & $\operatorname{Time}_{\text {Mission }}(\mathrm{min})$ & Range (nm) & $N O x(l b)$ \\
\hline Baseline & 0.785 & 35,000 & 0.637 & 488.0 & 3,485 & 306.7 \\
\hline Pareto M 1 & 0.770 & 36,500 & 0.628 & $500.5(+2.5 \%)$ & $3,516(+1.0 \%)$ & $298.5(-2.6 \%)$ \\
\hline Pareto M 4 & 0.755 & 36,500 & 0.622 & $507.6(+4.0 \%)$ & $3,511(+0.75 \%)$ & $290.0(-5.4 \%)$ \\
\hline Pareto M 5 & 0.750 & 36,500 & 0.620 & $509.7(+4.4 \%)$ & $3,508(+0.66 \%)$ & $288.0(-6.1 \%)$ \\
\hline Pareto M 6 & 0.745 & 36,500 & 0.618 & $511.4(+4.7 \%)$ & $3,503(+0.52 \%)$ & $285.7(-6.8 \%)$ \\
\hline Pareto $\mathrm{M} 8$ & 0.735 & 36,500 & 0.614 & $514.8(+5.5 \%)$ & $3,489(+0.1 \%)$ & $281.4(-8.2 \%)$ \\
\hline Pareto M20 & 0.700 & 31,500 & 0.612 & $522.8(+7.1 \%)$ & $(-2 \%)$ & $266.2(-13.2 \%)$ \\
\hline
\end{tabular}

Note: The optimisation is conducted to maximise Range (Fitness function 1) while minimising NOx (Fitness function 2) with fixed ramp weight $(174,670 \mathrm{lb})$ and the Time ${ }_{T_{-} \text {Mission }}$ represents the total mission time taken in minutes.

Figures 4 to 5 compare the total range, total mission fuel weight, total NOx, and aerodynamic performance (lift to drag ratio) obtained by the baseline flight conditions and Pareto optimal solutions along the mission. Figure 4 (a) compares the total range obtained by the baseline design and Pareto members $(1,4,56,8$, and 20). Pareto member 1 extends the range of the baseline aircraft by $1 \%$ while generating same amount of $\mathrm{CO}_{2}$ with less NOx emissions. Even though Pareto members $1-8$ have longer range, all Pareto members consume the same amount of mission fuel weight $(42,919 \mathrm{lb})$ as shown in Figure 4 (b). Figures 4 and 5 show that Pareto member 20 has 35 minutes longer flight time when compared to the baseline design however, it reduces NOx emission weight by $13.2 \%$. Figure 5 (b) compares the aerodynamic performance obtained by the Pareto optimal solutions and the baseline design; it can be seen that there is a 5\% aerodynamic improvement for Pareto member 20 when compared to the baseline condition. 


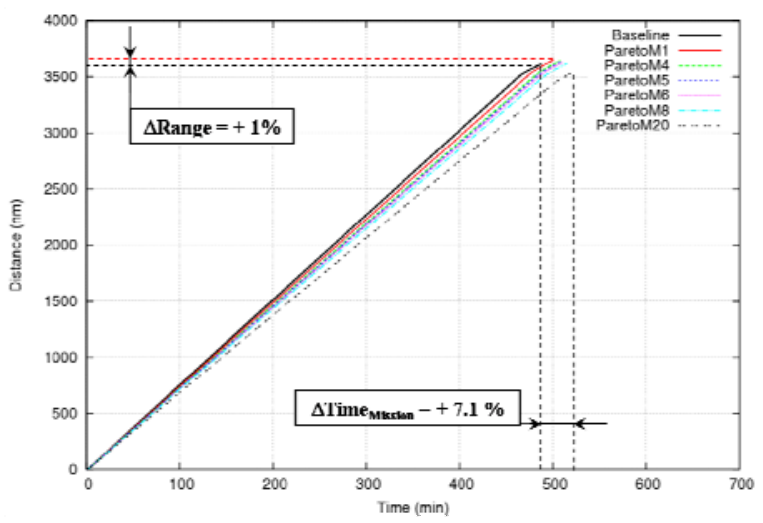

Figure 4 a). Range vs. Time.

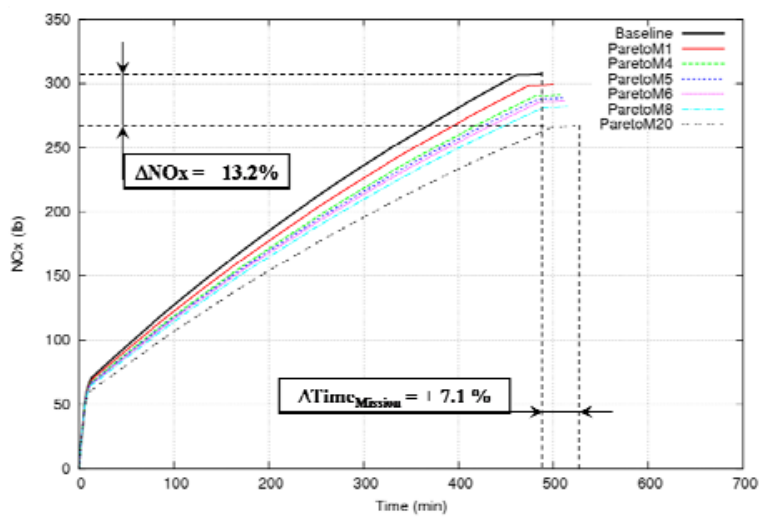

Figure 5 a). NOx vs. Time.

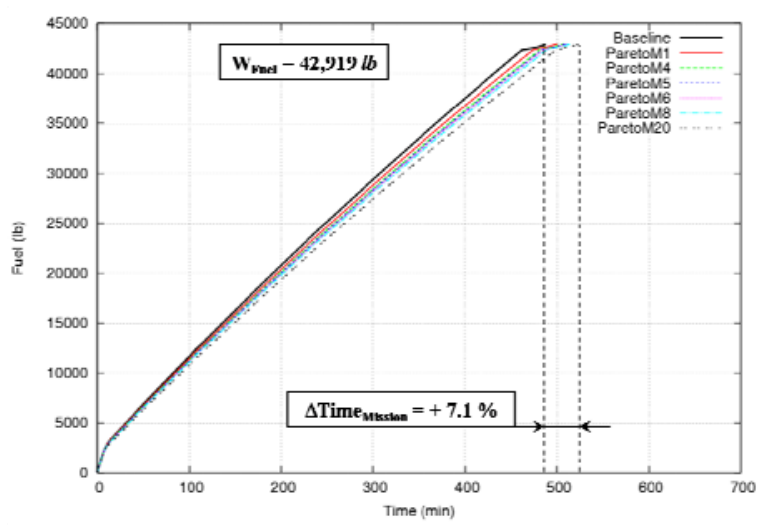

Figure 4 b). Fuel Weight vs. Time.

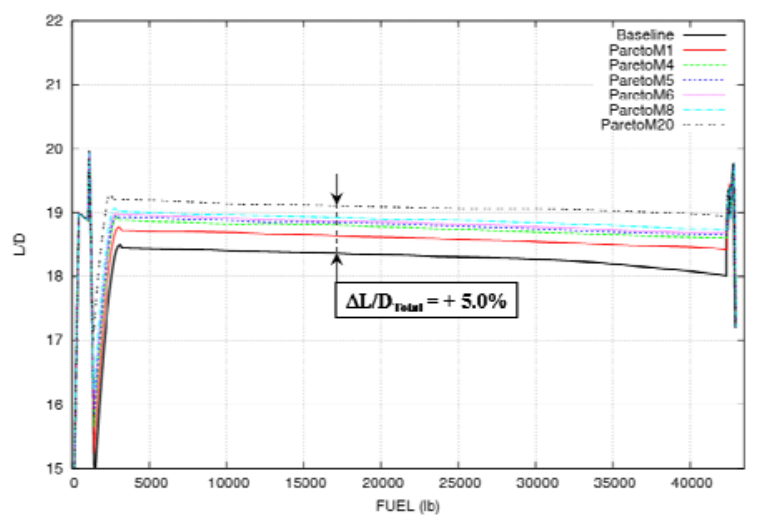

Figure 5 b). $L / D$ vs. Fuel Weight (right).

To conclude this optimisation test case, all Pareto members have a good trade-off in reducing NOx emissions. Pareto members 9-20 are good alternatives for the reduction of NOx emissions. In addition, it is also shown that Pareto members 1-8 are good solutions for extending the range and reducing NOx while consuming the same amount of fuel as the baseline conditions Mach 0.785 and altitude of $35,000 \mathrm{ft}$.

\subsection{Optimisation - II: Fixed Range}

\subsubsection{Problem Definition}

This optimisation consists of finding the best operating Mach and altitude conditions which allows the aircraft to have minimum mission fuel weight and NOx at fixed cruise range $(3,060 \mathrm{~nm})$. The fitness functions are defined by equations (1) and (2).

\subsubsection{Numerical Results}

The optimisation ran for 5 hours using one CPU $2.8 \mathrm{GHz}$. Pareto optimal solutions are illustrated in Figure 6 where all Pareto members produce lower NOx (fitness function 2) when compared to the baseline design. However Pareto members 9 to 20 are dominated by the baseline design in terms of the ratio of mission fuel weight to range (fitness function 1). Pareto members 1 to 8 can be selected as compromised solutions since they have lower mission fuel weight and NOx when compared to the baseline design as shown in Table 5. In other words, Pareto members 1 and 8 save operating cost while reducing the $\mathrm{CO}_{2}$ and NOx emissions. However the design engineers could choose one of the operating conditions obtained by Pareto members 9 to 20 due to NOx reduction if there is a regulation for NOx emission at the cruise conditions. 
Table 3. Mission Cruise Range and NOx obtained by optimal solutions and baseline design.

\begin{tabular}{ccccccc}
\hline Models & Mach & Altitude $(f t)$ & SFC & Time $_{\text {Mission }}(\mathrm{min})$ & $W_{\text {Fuel }}(\mathrm{lb})$ & NOx $(l b)$ \\
\hline Baseline & 0.785 & 35,000 & 0.637 & 431.4 & 37,206 & 265.8 \\
Pareto M 1 & 0.770 & 36,500 & 0.628 & $438.6(+1.6 \%)$ & $36,848(-1.0 \%)$ & $256.0(-4.0 \%)$ \\
Pareto M 4 & 0.755 & 36,500 & 0.622 & $447.5(+3.7 \%)$ & $36,934(-0.7 \%)$ & $247.5(-6.0 \%)$ \\
Pareto M 5 & 0.750 & 36,500 & 0.620 & $449.7(+4.2 \%)$ & $36,994(-0.6 \%)$ & $245.9(-7.0 \%)$ \\
Pareto M 6 & 0.745 & 36,500 & 0.618 & $452.0(+4.7 \%)$ & $37,064(-0.4 \%)$ & $244.5(-7.5 \%)$ \\
Pareto M 8 & 0.735 & 36,500 & 0.614 & $454.4(+5.3 \%)$ & $37,148(-0.2 \%)$ & $243.2(-8.5 \%)$ \\
Pareto M20 & 0.700 & 31,500 & 0.612 & $470.8(+9.1 \%)$ & $37,997(+2.0 \%)$ & $235.7(-11.3 \%)$ \\
\hline
\end{tabular}

Note: The optimisation is conducted to minimise $\mathrm{W}_{\text {Fuel }}$ (Fitness function 1) and NOx (Fitness function 2) with fixed cruise range $(3,060 \mathrm{~nm})$ and the Time $\mathrm{T}_{\mathrm{T}}$ Mission represents the total mission time taken.

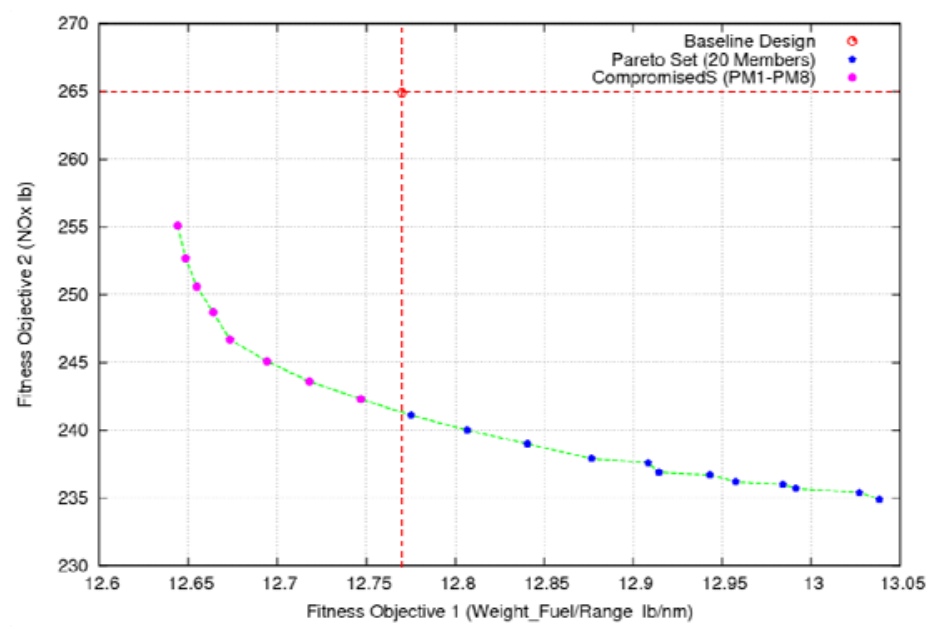

Figure 6. Pareto optimal front for NOx vs. $\mathrm{W}_{\text {Fuel }} /$ Range at fixed range weight.

Figures 7 to 8 show the total range, total mission fuel weight and total NOx, and aerodynamic performance (lift to drag ratio) for the baseline design and Pareto optimal solutions along the mission. Figure 7 (a) compares the total range obtained by the baseline design and Pareto members $(1,4,56,8$, and 20). The baseline aircraft can save $1 \%$ of total mission fuel weight at the operating conditions defined by Pareto member 1. Even though all Pareto members and the baseline design produce same mission range, Pareto members $(1,4,56,8)$ consume less amount of mission fuel weight as shown in Figure 7 (b). This means that Pareto members $(1,4,56,8)$ will produce less $\mathrm{CO}_{2}$ during the mission.

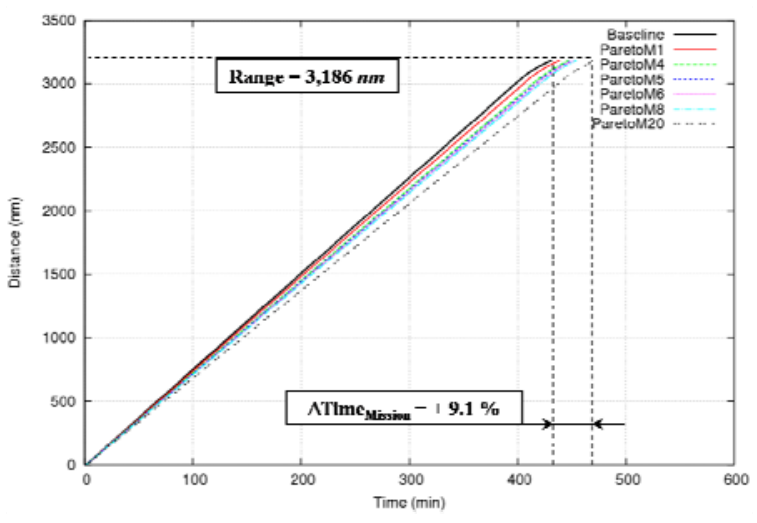

Figure 7 a). Range vs. Time.

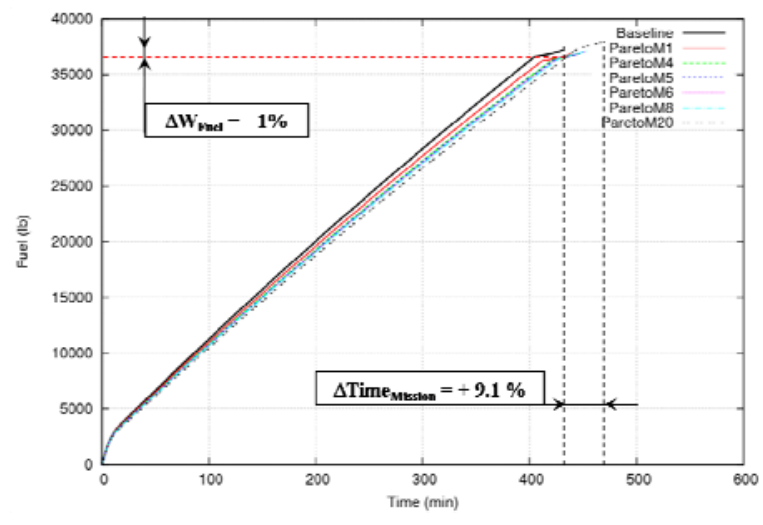

Figure 7 b). Fuel Weight vs. Time. 
Figure 8 (a) shows that Pareto member 20 has 39 minutes longer flight time when compared to the baseline design however, it produces $11.3 \%$ less NOx. In other words, the baseline aircraft can flight same distance while generating less amount of $\mathrm{CO}_{2}$ and $\mathrm{NOx}$ as well as less operating cost. Figure 8 (b) compares the aerodynamic performance obtained by the Pareto optimal solutions and the baseline design flight conditions; it can be seen that there is a 5\% aerodynamic improvement for Pareto member 20 when compared to the baseline conditions.

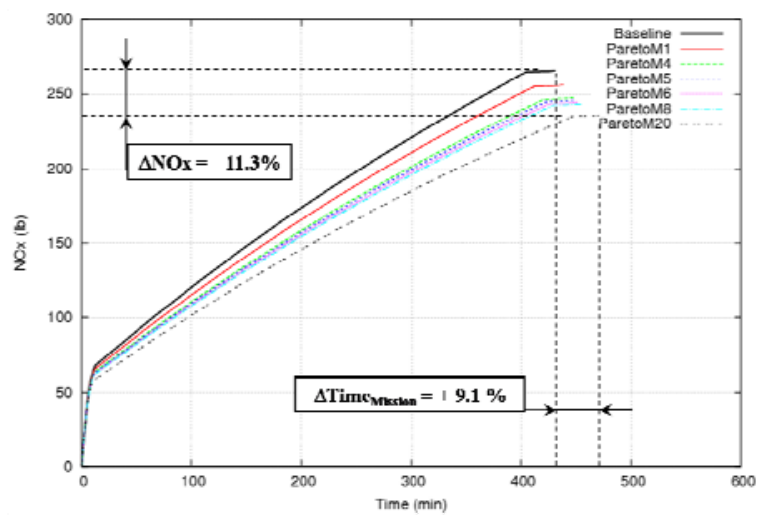

Figure 8 a). NOx vs. Time.

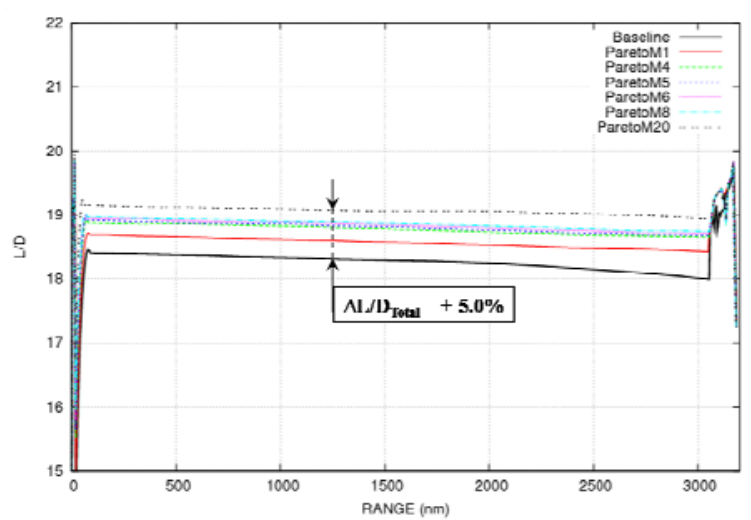

Figure 8 b). $L / D$ vs. Fuel Weight (right).

To conclude this optimisation, all Pareto members have a good trade-off in reducing NOx emissions. Pareto members $1-8$ are good solutions since they reduce NOx emission and the mission fuel weight $\left(\mathrm{CO}_{2}\right)$ while having the same range as the baseline flight conditions. Pareto members $9-20$ are also good solutions for the reduction of NOx emission while they have slightly higher mission fuel weight.

\section{Conclusion}

In this project, the analysis of range, mission fuel weight $\left(\mathrm{CO}_{2}\right)$ and $\mathrm{NOx}$ emission produced by a commercial aircraft operating at different Mach and altitude conditions has been described and investigated. The methodology couples a robust multi-objective evolutionary algorithm (HAPMOEA) and the multidisciplinary flight optimisation tool; FLOPS. Analytical research shows it to be a robust method to find optimum operating conditions for maximum range, minimum mission fuel weight $\left(\mathrm{CO}_{2}\right)$ and NOx without reconfiguring aircraft geometry or engine. Two practical design problems were studied and showed the broad applicability of method. A family of Pareto optimal design obtained from optimisation give the designer a selection so that they may proceed into more detail phases of the design process. Numerical results show that there is a limit to improve the range, mission fuel weight and NOx emission. This is because the optimisation is conducted without considering aircraft geometry or modifying engine configurations. Future work will focus on the extension studies by introducing changes of aircraft geometry and engine type with uncertainties in operating conditions such as Mach and altitude which are considered in this project.

\section{Acknowledgement}

The authors gratefully acknowledge Eric J. Whitney, and M. Sefrioui, Dassault Aviation for fruitful discussions on Hierarchical EAs and their contribution to the optimisation procedure and, also to L. A. McCullers at NASA for providing FLOPS software. The first and second authors would like to acknowledge the support the Australian Government's Cooperative Research Centres Program. 


\section{References}

[1] Sausen, R. and Schumann, U., Estimates of the Climate Response to Aircraft $\mathrm{CO}_{2}$ and NOx Emissions Scenarios, Climatic Change, Vol. 44, pp. 27-58, 2000.

[2] Penner, J., Aviation and the Global Atmosphere, Cambridge University Press.

[3] Gauss, M. Isaksen, I. S. A. and Sovde, O. A., Lee, D. S. Impact of Aircraft NOx Emissions on the Atmosphere - Tradeoffs to Reduce the Impact, Atmosphere Chemistry and Physics, Vol 6. Iss. 6, pp. 1529-1548, 2006.

[4] Schumann, U. The Impact Of Nitrogen Oxides Emissions From Aircraft Upon The Atmosphere At Flight Altitudes - Results From The AERONOX Project, Atmosphere Environment, Vol. 31, No. 12, pp. 1723-1733, 1997.

[5] McCullers L. A., FLOPS Flight Optimization System Release 6.02 User Guide, NASA Langley Research Center, 11 Mach, 2003.

[6] Geiselhart, K. A., A Technique for Integrating Engine Cycle and Aircraft Configuration Optimization. NASA CR-191602, February 1994.

[7] Geiselhart, K. A., Caddy, M. J., Morris S. J. Jr., Computer Program for Estimating Performance of Air-Breathing Aircraft Engines. NASA TM 4254, May 1991.

[8] Caddy, M. J., Shapiro, S. R. NEPCOMP - The Navy Engine Performance Computer Program, Version I. NADC-74045-30, April 1975.

[9] Goldberg, D., Genetic Algorithms in Search, Optimization and Machine Learning, AddisonWesley, 1989.

[10] Koza, J., Genetic Programming II. Massachusetts Institute of Technology, 1994.

[11] Michalewicz, Z., Genetic Algorithms + Data Structures = Evolution Programs. Artificial Intelligence, Springer-Verlag, 1992.

[12] Hansen, N. and Ostermeier, A., Completely Derandomized Self-Adaptation in Evolution Strategies. Evolutionary Computation, 9(2), pp. 159-195, 2001.

[13] Lee, D. S., Gonzalez, L. F. and Whitney, E. J., Multi-objective, Multidisciplinary Multi-fidelity Design tool: HAPMOEA - User Guide. 2007.

[14] Lee, D. S., Gonzalez, L. F., Periaux, J., and Srinivas, K., Evolutionary Optimisation Methods with Uncertainty for Modern Multidisciplinary Design in Aerospace Engineering, 100 Volumes of 'Notes on Numerical Fluid Mechanics' Heidelberg: Springer-Berlin, ISBN 978-3-540-708049, pages 271-284, Ch. 3. 2009.

[15] Lee, D. S., Gonzalez, L. F., Periaux, J., and Srinivas, K., Hybrid-Game Strategies Coupled to Evolutionary Algorithms for Robust Multidisciplinary Design Optimisation in Aerospace Engineering. IEEE Trans. Evolutionary Computation, TEVC-00213-2009. (Accepted)

[16] Goel, V., Contrails Avoidance With Flight Optimisation and Redesigning of Aircraft, Cranfield University, August 2007.

[17] Boeing, 737 Model Orders and Deliveries Data, Boeing, September, 2009.

[18] Boeing, 737 Family: 737-800 Technical Characteristics, http://www.boeing.com/commercial/737family/pf/pf 800tech.html.

[19] UIUC Airfoil Coordinates Database, http://www.ae.uiuc.edu/m-selig/ads/coord database.html.

[20] CFM, The Power Of Flight: CFM56-7B Technology, http://www.cfm56.com/products/cfm56$7 \mathrm{~b} / 9660$.

[21] The 737 Technical Site, Detailed Technical Data, http://www.b737.org.uk/techspecsdetailed.htm. 